\title{
Upper Semicontinuity of Attractors for a Non-Newtonian Fluid under Small Random Perturbations
}

\author{
Jianxin Luo \\ Oil and Gas Engineering Institute, Southwest Petroleum University, Chengdu, Sichuan 610500, China \\ Correspondence should be addressed to Jianxin Luo; luojianxin2014@163.com
}

Received 9 March 2014; Revised 11 May 2014; Accepted 15 May 2014; Published 19 June 2014

Academic Editor: Antonio Suárez

Copyright (C) 2014 Jianxin Luo. This is an open access article distributed under the Creative Commons Attribution License, which permits unrestricted use, distribution, and reproduction in any medium, provided the original work is properly cited.

This paper investigates the limiting behavior of attractors for a two-dimensional incompressible non-Newtonian fluid under small random perturbations. Under certain conditions, the upper semicontinuity of the attractors for diminishing perturbations is shown.

\section{Introduction}

Fluid flows arise in numerous scientific and industrial endeavors such as aeronautical sciences, meteorology, thermohydraulics, petroleum industry, and plasma physics. The equations describing the motion of the fluid flows are determined by its extra stress tensor. If the extra stress tensor of the fluid depends linearly on its symmetric part of the velocity gradient, the fluid is called Newtonian. Otherwise, the fluid is called non-Newtonian [1]. For instance, gases, water, motor oil, alcohols, and simple hydrocarbon compounds tend to be Newtonian fluids and their motions can be described by the Navier-Stokes equations. Molten plastics, polymer solutions, and paints tend to be non-Newtonian fluids, which may be described by the following system:

$$
\begin{aligned}
\frac{\partial u}{\partial t}+(u \cdot \nabla) u+\nabla p & =\nabla \cdot \tau(e(u))+g, \\
\nabla \cdot u & =0
\end{aligned}
$$

where the vector function $u(x, t)$ is the velocity of the fluid, $g$ is the external force, the scalar function $p$ represents the pressure, and $\tau(e(u))=\left(\tau_{i j}(e(u))\right)$ is the extra stress tensor of the fluid.

Ladyzhenskaya [2] formulated a two-dimensional nonNewtonian fluid model with the extra stress tensor:

$$
\tau_{i j}(e(u))=2 \mu_{0}\left(\varepsilon+|e|^{2}\right)^{-\alpha / 2} e_{i j}-2 \mu_{1} \Delta e_{i j}, \quad i, j=1,2,
$$

where

$$
e_{i j}=e_{i j}(u)=\frac{1}{2}\left(\frac{\partial u_{i}}{\partial x_{j}}+\frac{\partial u_{j}}{\partial x_{i}}\right), \quad|e|^{2}=\sum_{i, j=1}^{2}\left|e_{i j}\right|^{2},
$$

and $\mu_{0}, \mu_{1}, \alpha$, and $\varepsilon$ are parameters associated with the fluid and generally depend on temperature and pressure. The initial-boundary value problem of (1)-(2) on a 2D bounded domain $\mathbb{D}$ (with regular boundary) can be formulated as follows:

$$
\begin{aligned}
& \frac{\partial u}{\partial t}+(u \cdot \nabla) u+\nabla p \\
&= \nabla \cdot\left(2 \mu_{0}\left(\varepsilon+|e|^{2}\right)^{-\alpha / 2} e-2 \mu_{1} \Delta e\right)+g, \\
& \nabla \cdot u=0, \quad x \in \mathbb{D}, \\
& u=0, \quad \tau_{i j l} n_{j} n_{l}=0, \quad x \in \partial \mathbb{D}, \\
&\left.u\right|_{t=0}=u_{0},
\end{aligned}
$$

where $\tau_{i j l}=2 \mu_{1}\left(\partial e_{i j} / \partial x_{l}\right)(i, j, l=1,2)$ and $\mathbf{n}=\left(n_{1}, n_{2}\right)$ denotes the exterior unit normal vector to the boundary $\partial \mathbb{D}$. The first condition in (6) is the usual no-slip condition, while the second one expresses the fact that the first moments of the traction vanish on the boundary. This is a direct consequence of the principle of virtual work. We refer to [1-7] for more physical background. Recent results on well posedness, regularity, and long-term behavior of solutions for (4)-(7) are in, for example, [1-5, 7-10]. 
Attractors are an important concept in the study of infinite dimensional dynamical systems. There are numerous works on the autonomous and nonautonomous equations concerning this subject; see, for example, Chepyzhov and Vishik [11], Hale [12], Robinson [13], and Temam [14]. However, external forces or time-dependent influences in some fluid and materials phenomenon lead to the presence of stochastic terms in the above model equations (see, e.g., $[15,16])$.

The fundamental theory of random dynamical systems (RDS) was developed in 1990s by many people, including Arnold [15], Crauel and Flandoli [17], Crauel et al. [18], Flandoli and Schmalfuss [19], Caraballo, Langa, Robinson, and their coauthors [20-24].

The motivation for the present paper is the desire to understand the stability of attractors for the above twodimensional non-Newtonian fluid under vanishing small random perturbations.

We investigate the relations between the random attractor and its deterministic counterpart when the incompressible non-Newtonian fluid is subject to a small random perturbation, whose strength is measured by a small positive parameter $\epsilon$. Consider the problem (4)-(7) and the following $2 \mathrm{D}$ incompressible non-Newtonian fluid with an additive noise:

$$
\begin{aligned}
\mathrm{d} u_{\epsilon}= & \left\{-\left(u_{\epsilon} \cdot \nabla\right) u_{\epsilon}-\nabla p\right. \\
& \left.+\nabla \cdot\left[2 \mu_{0}\left(\varepsilon+\left|e_{\epsilon}\right|^{2}\right)^{-\alpha / 2} e_{\epsilon}-2 \mu_{1} \Delta e_{\epsilon}\right]+g\right\} \mathrm{d} t \\
+ & \epsilon \phi \mathrm{d} \omega(t), \\
& \nabla \cdot u_{\epsilon}=0, \quad x \in \mathbb{D} \subset \mathbb{R}^{2},
\end{aligned}
$$

where $e_{\epsilon}=\left(e_{\epsilon}\right)_{i j}=(1 / 2)\left(\left(\partial\left(u_{\epsilon}\right)_{i} / \partial x_{j}\right)+\left(\partial\left(u_{\epsilon}\right)_{j} / \partial x_{i}\right)\right)$, $\omega(t)$ is an independent two-sided Wiener processes, and $\phi$ is a function satisfying some conditions to be specified below. Caraballo et al. [25-28] proved the stability of the attractors for a class of evolution equations under small random perturbations and the results were applied to various physical equations. We note that (4) is regarded as the modified Navier-Stokes equations as the gradient $|\nabla u|$ of the velocity is relatively large [2]. Clearly, (4) reduces to NavierStokes equations when $\alpha=\mu_{1}=0$.

The main result in the present paper is the stability of the attractor in the sense that

$$
\lim _{\epsilon \rightarrow 0^{+}} \operatorname{dist}_{H}\left(\mathscr{A}_{\epsilon}, \mathscr{A}_{0}\right)=0 \quad \text { with probability one, }
$$

where $\operatorname{dist}_{H}(\cdot, \cdot)$ is the Hausdorff semidistance on the metric space $H$ (see notation in Section 2) and $\mathscr{A}_{\epsilon}$ and $\mathscr{A}_{0}$ are the attractors associated with (8) and (4)-(7), respectively. Given a $\delta>0$, we prove that there exists an $\epsilon_{0}$ (depending on $\omega$, a parameter event in a probability space $(\Omega, \mathscr{F}, \mathbb{P}))$ sufficiently small, such that the random attractors $\mathscr{A}_{\epsilon}$ are inside the $\delta$ neighborhood of the global attractor $\mathscr{A}_{0}$ for all $\epsilon \in\left(0, \epsilon_{0}\right)$ with probability one.

The paper is organized as follows. In the next section, we introduce some notations and recall some results from $[8,10]$.
Section 3 is devoted to prove the stability of solutions of the perturbed random system to the unperturbed deterministic system and then show the stability of the random attractor by showing $\lim _{\epsilon \rightarrow 0^{+}} \operatorname{dist}_{H}\left(\mathscr{A}_{\epsilon}(\omega), \mathscr{A}_{0}\right)=0$ with probability one.

\section{Global Existence and Uniqueness of Solutions}

In this section, we introduce some notations and recall some results about non-Newtonian fluid dynamics. Define

$$
\begin{aligned}
& \mathscr{V}=\left\{\varphi=\left(\varphi_{1}, \varphi_{2}\right) \in\left(\mathscr{C}_{0}^{\infty}(\overline{\mathbb{D}})\right)^{2}, \nabla \cdot \varphi=0 \text { in } \mathbb{D}, \varphi=\right. \\
& 0 \text { on } \partial \mathbb{D}\}, \\
& H=\text { closure of } \mathscr{V} \text { in }\left(L^{2}(\mathbb{D})\right)^{2} \text { with norm }\|\cdot\| \doteq \\
& \|\cdot\|_{\left(L^{2}(\mathbb{D})\right)^{2}} \\
& V=\text { closure of } \mathscr{V} \text { in }\left(H^{2}(\mathbb{D})\right)^{2} \text { with norm }\|\cdot\|_{V} \doteq \\
& \|\cdot\|_{\left(H^{2}(\mathbb{D})\right)^{2}}, \\
& H^{\prime}=\text { dual space of } H, V^{\prime}=\text { dual space of } V,
\end{aligned}
$$

where $(\cdot, \cdot)$ denotes the inner product in $H$ and $\langle\cdot, \cdot\rangle$ stands for the dual pairing between $V$ and $V^{\prime}$. If we identify $H$ with $H^{\prime}$, then $V \hookrightarrow H=H^{\prime} \hookrightarrow V^{\prime}$ with continuous and compact embeddings.

We also define the bilinear form

$$
a(u, v)=\sum_{i, j, k=1}^{2} \int_{\mathbb{D}} \frac{\partial e_{i j}(u)}{\partial x_{k}} \frac{\partial e_{i j}(v)}{\partial x_{k}} \mathrm{~d} x, \quad u, v \in V .
$$

Lemma 1 (see [4]). There exist two positive constants $c_{1}$ and $c_{2}$, which depend only on $\mathbb{D}$, such that

$$
c_{1}\|u\|_{V}^{2} \leq a(u, u) \leq c_{2}\|u\|_{V}^{2}, \quad \forall u \in V
$$

From the definition of $a(\cdot, \cdot)$ and Lemma 1 we see that $a(\cdot, \cdot)$ defines a positive definite symmetric bilinear form on $V$. As a consequence of the Lax-Milgram Lemma, we obtain an operator $A \in \mathscr{L}\left(V, V^{\prime}\right)$, via

$$
\langle A u, v\rangle=a(u, v), \quad \forall u, v \in V .
$$

Moreover, let $D(A)=\{u \in V: A u \in H\}$, and then $D(A)$ is a Hilbert space. We have (see [8])

$$
c_{1}\|u\|_{V} \leq\|A u\| \text {. }
$$

For brevity, we use $H_{0}^{1}(\mathbb{D})$ to denote $\left(H_{0}^{1}(\mathbb{D})\right)^{2}$ in the sequel. We also define a continuous trilinear form on $H_{0}^{1}(\mathbb{D}) \times$ $H_{0}^{1}(\mathbb{D}) \times H_{0}^{1}(\mathbb{D})$ as follows:

$$
b(u, v, w)=\sum_{i, j=1}^{2} \int_{\mathbb{D}} u_{i} \frac{\partial v_{j}}{\partial x_{i}} w_{j} \mathrm{~d} x, \quad u, v, w \in H_{0}^{1}(\mathbb{D}) .
$$

Since $V \subset H_{0}^{1}(\mathbb{D}), b(\cdot, \cdot, \cdot)$ is continuous on $V \times V \times V$ and one can check

$$
b(u, v, w)=-b(u, w, v), \quad b(u, v, v)=0, \quad \forall u, v, w \in V .
$$


Now for any $u \in V$,

$$
\langle B(u, u), w\rangle=b(u, u, w), \quad \forall w \in V,
$$

defines a continuous functional $B(u)$ from $V \times V$ to $V^{\prime}$. Finally, for $u \in V$, we set $\mu(u)=2 \mu_{0}\left(\varepsilon+|e(u)|^{2}\right)^{-\alpha / 2}$ and define $N(u)$ as

$$
\langle N(u), v\rangle=\sum_{i, j=1}^{2} \int_{\mathbb{D}} \mu(u) e_{i j}(u) e_{i j}(v) \mathrm{d} x, \quad \forall v \in V .
$$

Then the functional $N(u)$ is continuous from $V$ to $V^{\prime}$. When $u \in D(A), N(u)$ can be extended to $H$ via

$$
\langle N(u), v\rangle=-\int_{\mathbb{D}}\{\nabla \cdot[\mu(u) e(u)]\} \cdot v \mathrm{~d} x, \quad \forall v \in H .
$$

Eliminating the pressure $p$ by a proper projection, we have the weak version of problem (4)-(7) in the solenoidal vector fields as follows (see $[4,9])$ :

$$
\begin{gathered}
\frac{\partial u}{\partial t}+2 \mu_{1} A u+B(u, u)+N(u)=g, \\
\left.u\right|_{t=0}=u_{0} .
\end{gathered}
$$

Lemma 2 (see $[8,9])$. Let $g \in H$ (independent of time $t$ ). Then the semigroup $\{S(t)\}_{t \geq 0}$ associated with (19) possesses a global attractor $\mathscr{A}_{0}$ in $H$ satisfying

(1) (compactness) $\mathscr{A}_{0}$ is compact in $H$;

(2) (invariance) $S(t) \mathscr{A}_{0}=\mathscr{A}_{0}, t \geq 0$;

(3) (attractivity) for all $\mathscr{B} \subset \subset \quad H$ bounded, $\lim _{t \rightarrow+\infty} \operatorname{dist}_{H}\left(S(t) \mathscr{B}, \mathscr{A}_{0}\right)=0$.

Moreover, $\mathscr{A}_{0}$ is compact in space $V$ and for any $\mathscr{B}^{V} \subset V$ bounded,

$$
\lim _{t \rightarrow+\infty} \operatorname{dist}_{V}\left(S(t) \mathscr{B}^{V}, \mathscr{A}_{0}\right)=0 \text {. }
$$

In this paper, we use the concepts concerning the metric dynamical system (MDS), random dynamical system (RDS), random closed set, and global random attractor from [15].

We now take $\Omega=\{\omega \in \mathscr{C}(\mathbb{R} ; H), \omega(0)=0\}$ and endow it with the compact open topology (see Appendices A.2 and A.3 in [15]). Take $\mathbb{P}$ as the corresponding product measure of two Wiener measures on the negative and the positive time parts of $\Omega$, and denote $\mathscr{F}_{0}$ by the Borel $\sigma$-algebra on $\Omega$. Let

$$
\theta_{t} \omega(s)=\omega(s+t)-\omega(t), \quad t, s \in \mathbb{R} .
$$

To obtain the uniqueness and existence of solutions for the problem (8), we need the following assumption.

Assumption A. If $\mu_{0}, \mu_{1}, \varepsilon>0, \alpha \in(0,1), g \in H$, and $\phi \in$ $D(A)$, then there exists a positive number $\delta$ such that

$$
|\langle B(u, \phi), u\rangle| \leq \delta\|u\|^{2}, \quad \forall u \in V .
$$

Remark 3. Since $\langle B(u, \phi), u\rangle=\sum_{i, j=1}^{2} \int_{\mathbb{D}} u_{i}\left(\partial \phi_{j} / \partial x_{i}\right) u_{j} \mathrm{~d} x$, we see that the assumption (22) is satisfied provided that the function $\phi$ is Lipschitz continuous on $\mathbb{D}$.
Using the notations and operators introduced above, we can put (8) into the following abstract form:

$$
\mathrm{d} u_{\epsilon}=\left[-2 \mu_{1} A u_{\epsilon}-B\left(u_{\epsilon}, u_{\epsilon}\right)-N\left(u_{\epsilon}\right)+g\right] \mathrm{d} t+\epsilon \phi \mathrm{d} \omega(t),
$$

$$
\left.u_{\epsilon}\right|_{t=0}=u_{0} \text {. }
$$

Let $\eta>0$ be a constant and denote $z$ by the solution of the stationary solution of the Itô equation:

$$
\mathrm{d} z=-\eta z \mathrm{~d} t+\mathrm{d} \omega(t) .
$$

The solution is often called an Ornstein-Uhlenbeck process. In fact,

$$
z(t)=\int_{-\infty}^{t} e^{-\eta(t-s)} \mathrm{d} \omega(s)
$$

We now make the change

$$
v_{\epsilon}(t, \omega)=u_{\epsilon}(t, \omega)-\epsilon \phi z(t, \omega) .
$$

Then $v_{\epsilon}(t, \omega)$ satisfies the following random abstract evolutionary equation:

$$
\begin{gathered}
\frac{\mathrm{d} v_{\epsilon}}{\mathrm{d} t}+2 \mu_{1} A v_{\epsilon}+B\left(u_{\epsilon}, u_{\epsilon}\right)+N\left(u_{\epsilon}\right) \\
+\epsilon \eta \phi z+2 \mu_{1} \epsilon A(\phi z)=g .
\end{gathered}
$$

Now (28) can be studied for each $\omega \in \Omega$.

Lemma 4 (see [10]). Let Assumption A hold. Then for $\mathbb{P}$-a.s. $\omega \in \Omega$, the following results hold.

(1) For all $t_{0} \in \mathbb{R}$ and $v_{0}=v_{\epsilon}(0) \in H$, there exists a unique solution $v_{\epsilon} \in \mathscr{C}\left(\left[t_{0},+\infty\right) ; H\right) \cap L_{\text {loc }}^{2}\left(t_{0},+\infty ; V\right)$ of $(28)$ with initial value $v_{0}$.

(2) If $v_{0} \in V$, then the solution belongs to $\mathscr{C}\left(\left[t_{0},+\infty\right) ; V\right) \cap$ $L_{\text {loc }}^{2}\left(t_{0},+\infty ; D(A)\right)$.

(3) For every $\tau>0, v_{\epsilon}(t) \in \mathscr{C}\left(\left[t_{0}+\tau,+\infty\right)\right.$; $\left.V\right) \cap L_{\text {loc }}^{2}\left(t_{0}+\right.$ $\tau,+\infty ; D(A))$.

(4) Denote the solution by $v_{\epsilon}\left(t, \omega ; t_{0}, v_{0}\right)$. Then the map $v_{0} \mapsto v_{\epsilon}\left(t, \omega ; t_{0}, v_{0}\right)$ is continuous for all $t \geq t_{0}$.

By Lemma 4 we see that for each $\epsilon \in(0,1]$ there is a continuous mapping from $H$ into itself: $v_{0} \mapsto v_{\epsilon}\left(t, \omega ; t_{0}, v_{0}\right)$, where $v_{\epsilon}\left(t, \omega ; t_{0}, v_{0}\right)$ is the solution of (28) with initial value $v_{\epsilon}\left(t_{0}\right)=v_{0}$. Thus for each $\epsilon \in(0,1]$, we can define an RDS $\psi_{\epsilon}(t, \omega)$ associated with (23) and (24) in $H$ by setting

$$
\begin{aligned}
\psi_{\epsilon}(t, \omega) u_{0} & =u_{\epsilon}(t, \omega)=v_{\epsilon}(t, \omega)+\epsilon \phi z(t, \omega) \\
& =v_{\epsilon}\left(t, \omega ; 0, u_{0}-\epsilon \phi z(0, \omega)\right)+\epsilon \phi z(t, \omega) .
\end{aligned}
$$

We end this section with the concept of random attractor for $\psi_{\epsilon}(t, \omega)$ in space $H$.

Definition 5. Let the RDS $\{\psi(t, \omega)\}_{t \geq 0, \omega \in \Omega}$ on $(\Omega, \mathscr{F}, \mathbb{P}$, $\left.\left(\theta_{t}\right)_{t \in \mathbb{R}}\right)$ be defined by (29) with state space $H$, if $\omega \mapsto \mathscr{A}(\omega)$ satisfies the following: 
(i) (random compactness) $\mathscr{A}(\omega)$ is a random compact set of $H$ for a.e. $\omega \in \Omega$,

(ii) (invariance) for a.e. $\omega \in \Omega$ and all $t \geq 0, S(t, \omega) \mathscr{A}(\omega)=$ $\mathscr{A}\left(\theta_{t} \omega\right)$,

(iii) (attracting property) for any bounded subset $\mathscr{B}$ of $H$ and a.e. $\omega \in \Omega$,

$$
\lim _{t \rightarrow+\infty} \operatorname{Dist}_{H}\left(S\left(t, \theta_{-t} \omega\right) \mathscr{B}, \mathscr{A}(\omega)\right)=0 .
$$

Then $\mathscr{A}(\omega)$ is called a global random attractor for $\{\psi(t, \omega)\}_{t \geq 0, \omega \in \Omega}$ in space $H$.

\section{Stability of Attractors}

We first prove the following lemma, which plays a key role later on.

Lemma 6. The solution $u_{\epsilon}\left(0, \omega ;-t_{0}, u_{0}\right)$ of (23) and (24) converges in $H \mathbb{P}$-a.s. as $\epsilon \rightarrow 0^{+}$to the solution $u\left(t_{0} ; u_{0}\right)$ of the unperturbed problem (19), uniformly on bounded sets of initial conditions. That is, for $\mathbb{P}$ almost every $\omega \in \Omega, t_{0} \in \mathbb{R}_{+}$and $\mathscr{B} \subset H$ bounded, the following convergence holds:

$$
\lim _{\epsilon \rightarrow 0^{+}}\left\|u_{\epsilon}\left(0, \omega ;-t_{0}, u_{0}\right)-u\left(t_{0}, u_{0}\right)\right\|=0 .
$$

Proof. Let $w_{\epsilon}(t, \omega)=u_{\epsilon}(t, \omega)-u(t)$ be the difference between the solutions of the perturbed and unperturbed equations with the same initial condition $u_{0}$ at $-t_{0}$. It is clear that $w_{\epsilon}$ satisfies

$$
\begin{gathered}
\mathrm{d} w_{\epsilon}+\left[2 \mu_{1} A w_{\epsilon}+B\left(u_{\epsilon}, u_{\epsilon}\right)-B(u, u)\right. \\
\left.+N\left(u_{\epsilon}\right)-N(u)\right] \mathrm{d} t=\epsilon \phi \mathrm{d} \omega(t), \\
w_{\epsilon}\left(-t_{0}\right)=0 .
\end{gathered}
$$

We now make the change of variables

$$
z_{\epsilon}=w_{\epsilon}-\epsilon \phi \omega(t)
$$

and thus formally obtain

$$
\begin{gathered}
\frac{\mathrm{d} z_{\epsilon}}{\mathrm{d} t}+2 \mu_{1} A z_{\epsilon}+2 \mu_{1} \epsilon A(\phi \omega(t))+B\left(u_{\epsilon}, u_{\epsilon}\right) \\
-B(u, u)+N\left(u_{\epsilon}\right)-N(u)=0 .
\end{gathered}
$$

Taking the inner product of the above equation with $z_{\epsilon}$, we get

$$
\begin{aligned}
& \frac{1}{2} \frac{\mathrm{d}}{\mathrm{d} t}\left\|z_{\epsilon}\right\|^{2}+2 \mu_{1}\left(A z_{\epsilon}, z_{\epsilon}\right)+2 \mu_{1} \epsilon\left(\omega(t) A \phi, z_{\epsilon}\right) \\
& \quad+\left\langle B\left(u_{\epsilon}, u_{\epsilon}\right), z_{\epsilon}\right\rangle-\left\langle B(u, u), z_{\epsilon}\right\rangle+\left\langle N\left(u_{\epsilon}\right), z_{\epsilon}\right\rangle \\
& \quad-\left\langle N(u), z_{\epsilon}\right\rangle=0 .
\end{aligned}
$$

Therefore

$$
\begin{aligned}
2 \mu_{1} \epsilon\left(\omega(t) A \phi, z_{\epsilon}\right) & \leq 2 \mu_{1} \epsilon|\omega(t)|\|A \phi\|\left\|z_{\epsilon}\right\| \\
& \leq 2 \mu_{1}^{2} \epsilon^{2} \omega^{2}(t)\|A \phi\|^{2}+\frac{\left\|z_{\epsilon}\right\|^{2}}{2} .
\end{aligned}
$$

By the property of the operator $B(\cdot, \cdot)$, we imply that

$$
\begin{aligned}
& B\left(u_{\epsilon}, u_{\epsilon}\right)-B(u, u) \\
&= B\left(w_{\epsilon}+u, w_{\epsilon}+u\right)-B(u, u) \\
&= B\left(w_{\epsilon}, w_{\epsilon}\right)+B\left(w_{\epsilon}, u\right)+B\left(u, w_{\epsilon}\right) \\
&= B\left(z_{\epsilon}+\epsilon \phi \omega(t), z_{\epsilon}+\epsilon \phi \omega(t)\right) \\
&+B\left(z_{\epsilon}+\epsilon \phi \omega(t), u\right)+B\left(u, z_{\epsilon}+\epsilon \phi \omega(t)\right) .
\end{aligned}
$$

Thus,

$$
\begin{aligned}
\left\langle B\left(u_{\epsilon}, u_{\epsilon}\right)-B(u, u), z_{\epsilon}\right\rangle & \\
= & \left\langle B\left(z_{\epsilon}+\epsilon \phi \omega(t), z_{\epsilon}+\epsilon \phi \omega(t)\right), z_{\epsilon}\right\rangle \\
& +\left\langle B\left(z_{\epsilon}+\epsilon \phi \omega(t), u\right), z_{\epsilon}\right\rangle+\left\langle B\left(u, z_{\epsilon}+\epsilon \phi \omega(t)\right), z_{\epsilon}\right\rangle \\
= & \left\langle B\left(z_{\epsilon}, z_{\epsilon}+\epsilon \phi \omega(t)\right), z_{\epsilon}\right\rangle+\left\langle B(\epsilon \phi \omega(t), \epsilon \phi \omega(t)), z_{\epsilon}\right\rangle \\
& +\left\langle B\left(z_{\epsilon}, u\right), z_{\epsilon}\right\rangle+\left\langle B(\epsilon \phi \omega(t), u), z_{\epsilon}\right\rangle \\
& +\left\langle B(u, \epsilon \phi \omega(t), u), z_{\epsilon}\right\rangle .
\end{aligned}
$$

We now estimate the right hand side of (38) terms by terms. First note that

$$
\begin{aligned}
& \left\langle B\left(z_{\epsilon}, \epsilon \phi \omega(t), z_{\epsilon}\right)\right\rangle \leq \epsilon|\omega(t)|\left|\left\langle B\left(z_{\epsilon}, \phi\right), z_{\epsilon}\right\rangle\right| \\
& \leq \delta \epsilon|\omega(t)|\left\|z_{\epsilon}\right\|^{2}, \\
& \left\langle B(\epsilon \phi \omega(t), \epsilon \phi \omega(t)), z_{\epsilon}\right\rangle \\
& \leq \epsilon^{2} \omega^{2}(t)\left\langle B(\phi, \phi), z_{\epsilon}\right\rangle \\
& \leq \epsilon^{2} \omega^{4}(2)\|\phi\|_{L^{4}(\mathbb{D})}\|\nabla \phi\|_{L^{4}(\mathbb{D})}\left\|z_{\epsilon}\right\| \\
& \leq c \epsilon^{2} \omega^{2}(t)\|\phi\|^{1 / 2}\|\nabla \phi\|^{1 / 2}\|\phi\|^{1 / 4}\|\Delta \phi\|^{3 / 4}\left\|z_{\epsilon}\right\| \\
& \leq c \epsilon^{2} \omega^{4}(t)\|\nabla \phi\|^{2}\|\Delta \phi\|^{2}+\frac{\left\|z_{\epsilon}\right\|^{2}}{2}, \\
& \left\langle B\left(z_{\epsilon}, u\right), z_{\epsilon}\right\rangle \leq c\left\|z_{\epsilon}\right\|\|\nabla u\|\left\|\nabla z_{\epsilon}\right\| \\
& \quad \leq \frac{3 \mu_{1}}{4}\left\|z_{\epsilon}\right\|_{V}^{2}+c\|\nabla u\|^{2}\left\|z_{\epsilon}\right\|^{2}, \\
& \left\langle B(\epsilon \phi \omega(t), u), z_{\epsilon}\right\rangle \\
& \leq \epsilon|\omega(t)|\left|\left\langle B(\phi, u), z_{\epsilon}\right\rangle\right|
\end{aligned}
$$




$$
\begin{aligned}
& \leq \epsilon|\omega(t)|\|\phi\|_{L^{4}(\mathbb{D})}\|\nabla u\|_{L^{4}(\mathbb{D})}\left\|z_{\epsilon}\right\| \\
& \leq \epsilon|\omega(t)|\|\phi\|^{1 / 2}\|\nabla \phi\|^{1 / 2}\|\nabla u\|^{1 / 2}\|\Delta u\|^{1 / 2}\left\|z_{\epsilon}\right\| \\
& \leq c \epsilon^{2}|\omega(t)|^{2}\|\phi\|\|\nabla \phi\|\|u\|_{V}^{2}+\frac{\left\|z_{\epsilon}\right\|^{2}}{2}, \\
& \left\langle B(u, \epsilon \phi \omega(t)), z_{\epsilon}\right\rangle \\
& \leq \epsilon|\omega(t)|\left|\left\langle B(u, \phi), z_{\epsilon}\right\rangle\right| \\
& \leq \epsilon|\omega(t)|\|u\|^{1 / 2}\left|\|\nabla u\|^{1 / 2}\right|\|\nabla \phi\|^{1 / 2}\|\Delta \phi\|^{1 / 2}\left\|z_{\epsilon}\right\| \\
& \leq \epsilon^{2}|\omega(t)|^{2}\|u\|\|u\|_{H^{1}(\mathbb{D})}^{2}\|\phi\|_{H^{1}(\mathbb{D})}\|A \phi\|+\frac{\left\|z_{\epsilon}\right\|^{2}}{2} .
\end{aligned}
$$

Then also note that

$$
\left\langle N\left(u_{\epsilon}\right)-N(u), z_{\epsilon}\right\rangle=\left\langle N\left(u_{\epsilon}\right)-N(u), u_{\epsilon}-u-\epsilon \phi \omega(t)\right\rangle .
$$

By the nonnegativity of $N(\cdot)$ (see [4]), we conclude that

$$
\left\langle N\left(u_{\epsilon}\right)-N(u), u_{\epsilon}-u\right\rangle \geq 0 .
$$

Set $F(s)=2 \mu_{0}\left(\varepsilon+|s|^{2}\right)^{-\alpha / 2} s$, where

$$
\begin{aligned}
s & =\left(\begin{array}{ll}
s_{1} & s_{2} \\
s_{3} & s_{4}
\end{array}\right) \in \mathscr{M}_{2 \times 2}, \\
|s|^{2} & =\sum_{i=1}^{4} s_{i}^{2}, \quad s_{i} \in \mathbb{R}, i=1,2,3,4 .
\end{aligned}
$$

Here $\mathscr{M}_{2 \times 2}$ denotes the matrix of order $2 \times 2$. Then the first order Fréchet derivative of $F(s)$ is

$\mathrm{DF}(s)$

$$
\begin{aligned}
=2 \mu_{0}\left(\varepsilon+|s|^{2}\right)^{-\alpha / 2} & \left(\begin{array}{cccc}
1-\frac{\alpha s_{1}^{2}}{\varepsilon+|s|^{2}} & -\frac{\alpha s_{1} s_{2}}{\varepsilon+|s|^{2}} & -\frac{\alpha s_{1} s_{3}}{\varepsilon+|s|^{2}} & -\frac{\alpha s_{1} s_{4}}{\varepsilon+|s|^{2}} \\
-\frac{\alpha s_{1} s_{2}}{\varepsilon+|s|^{2}} & 1-\frac{\alpha s_{2}^{2}}{\varepsilon+|s|^{2}} & -\frac{\alpha s_{2} s_{3}}{\varepsilon+|s|^{2}} & -\frac{\alpha s_{2} s_{4}}{\varepsilon+|s|^{2}} \\
-\frac{\alpha s_{1} s_{3}}{\varepsilon+|s|^{2}} & -\frac{\alpha s_{2} s_{3}}{\varepsilon+|s|^{2}} & 1-\frac{\alpha s_{3}^{2}}{\varepsilon+|s|^{2}} & -\frac{\alpha s_{3} s_{4}}{\varepsilon+|s|^{2}} \\
-\frac{\alpha s_{1} s_{4}}{\varepsilon+|s|^{2}} & -\frac{\alpha s_{2} s_{4}}{\varepsilon+|s|^{2}} & -\frac{\alpha s_{3} s_{4}}{\varepsilon+|s|^{2}} & 1-\frac{\alpha s_{4}^{2}}{\varepsilon+|s|^{2}}
\end{array}\right) .
\end{aligned}
$$

Since $0<\alpha<1$, we have

$$
\begin{aligned}
\left|-\frac{\alpha s_{i} s_{j}}{\varepsilon+|s|^{2}}\right| & <\left|\frac{s_{i} s_{j}}{\varepsilon+|s|^{2}}\right|<1, \quad i, j=1,2,3,4, \\
0 & <1-\frac{\alpha s_{i}^{2}}{\varepsilon+|s|^{2}}<1, \quad i=1,2,3,4 .
\end{aligned}
$$

Consequently,

$$
\|\mathrm{DF}(s)\| \leq 8 \mu_{0}\left(\varepsilon+|s|^{2}\right)^{-\alpha / 2} \leq 8 \mu_{0} \varepsilon^{-\alpha / 2}, \quad \forall s \in \mathscr{M}_{2 \times 2} .
$$

Now for any $a, b \in \mathbb{R}^{4}$,

$$
F(b)-F(a)=\int_{0}^{1} \mathrm{DF}(a+\tau(b-a))(b-a) \mathrm{d} \tau .
$$

Taking $a=e\left(u_{\epsilon}\right)=\left(e_{i j}\left(u_{\epsilon}\right)\right), b=e(u)=\left(e_{i j}(u)\right)$, applying the integration by parts first, and then utilizing the above inequality about $F(s)$, we have

$$
\begin{aligned}
& \left\langle N\left(u_{\epsilon}\right)-N(u), \epsilon \phi \omega(t)\right\rangle \\
& \quad=\sum_{i, j=1}^{2} \int_{\mathbb{D}}\left[F\left(e_{i j}\left(u_{\epsilon}\right)\right)-F\left(e_{i j}(u)\right)\right] \cdot e_{i j}(\epsilon \phi \omega(t)) \mathrm{d} x \\
& \quad \leq c \epsilon|\omega(t)|\left(\left\|\nabla\left(u_{\epsilon}-u\right)\right\|\right)\|\nabla \phi\| \\
& \quad \leq c \epsilon|\omega(t)|\left\|\nabla\left(z_{\epsilon}+\epsilon \phi \omega(t)\right)\right\|\|\nabla \phi\| \\
& \quad \leq \frac{3 \mu_{1}}{4}\left\|\nabla z_{\epsilon}\right\|^{2}+c \epsilon^{2} \omega^{2}(t)\|\nabla \phi\|^{2} .
\end{aligned}
$$

Combining (35)-(40) and (47), we obtain

$$
\frac{\mathrm{d}}{\mathrm{d} t}\left\|z_{\epsilon}\right\|^{2} \leq I(t)+G(t)\left\|z_{\epsilon}\right\|^{2}
$$

where

$$
\begin{aligned}
I(t)=c \epsilon[ & \mu_{1}^{2} \epsilon \omega^{2}(t)\|A \phi\|^{2}+\epsilon \omega^{4}(t)\|\nabla \phi\|^{2}\|A \phi\|^{2} \\
& +\epsilon|\omega(t)|\|\phi\|\|\nabla \phi\|\|u\|_{V}^{2}+\epsilon\left|\omega^{2}(t)\right|\|u\| \\
& \left.\times\|\nabla u\|\|\nabla \phi\|\|A \phi\|+\epsilon\left|\omega^{2}(t)\right|\|A \phi\|^{2}\right], \\
G(t)=2+ & \epsilon \delta|\omega(t)|+c\|\nabla u\|^{2} .
\end{aligned}
$$

Then, by Gronwall inequality,

$$
\left\|z_{\epsilon}(t)\right\|^{2} \leq I(t)+\int_{-t_{0}}^{t} I(s) G(s) \exp \left(\int_{s}^{t} G(\tau) \mathrm{d} \tau\right) \mathrm{d} s .
$$

By Lemma 2 we find that $\|u(t)\|_{V}^{2}$ is uniformly bounded with the initial values belonging to a bounded set (see [8] for details). Obviously, $\|u(t)\| \leq\|u(t)\|_{V},\|\nabla u(t)\| \leq\|u(t)\|_{V}$. Thus, $I(t) \rightarrow 0$ as $\epsilon \rightarrow 0^{+}$and by (50) we get $\left\|z_{\epsilon}(t)\right\|^{2} \rightarrow 0$ as $\epsilon \rightarrow 0^{+}$for all $t \geq-t_{0}$. Therefore,

$$
\lim _{\epsilon \rightarrow 0^{+}}\left\|w_{\epsilon}(t)\right\|^{2} \leq \lim _{\epsilon \rightarrow 0^{+}} 2\left(\left\|z_{\epsilon}\right\|^{2}+\epsilon^{2}\|\phi\| \omega^{2}(t)\right)=0 .
$$

We complete the proof by taking $t=0$.

Remark 7. The RDS (see (29)) is defined using the transformation (27) into a random equation with the OrnsteinUhlenbeck process. The estimates in Lemma 6 are obtained 
using a change of variable (33). It is just for the convenience of computation. In fact, we can check that the functions given by the two changes of variable satisfy the same equation (23). Thus the solutions $u_{\epsilon}$ of the stochastic system (23) given by the two changes of variables are the same.

Lemma 8. Let Assumption A hold. Then for each $\epsilon \in(0,1]$, there exists a random attractor $\mathscr{A}_{\epsilon}(\omega)$ for the $R D S \psi_{\epsilon}(t, \omega)$ associated with (23) and (24), and in addition there exists a compact set $\mathscr{K}$ such that $\mathbb{P}$-a.s.

$$
\lim _{\epsilon \rightarrow 0^{+}} \operatorname{dist}_{H}\left(\mathscr{A}_{\epsilon}(\omega), \mathscr{K}\right)=0
$$

Proof. For each $\epsilon \in(0,1]$, the existence of a random attractor $\mathscr{A}_{\epsilon}(\omega)$ for the RDS $\psi_{\epsilon}(t, \omega)$ associated with (23) and (24) can be found in [10]. Also, the existence of a compact set $\mathscr{K}$ and (52) can be established, with a similar way to Lemma 4.3 of [10] by showing that $\|u(0)\|_{V} \leq r(\omega)$ (the radius of random absorbing set in $V$ ) and $\lim _{\epsilon \rightarrow 0^{+}} r(\omega) \leq r_{d}$ with $r_{d}$ independent of $\omega \in \Omega$. The detailed proof is omitted here.

By Lemmas 3.1 and 3.3 and Theorem 2 of [25] and Theorem 1.1 of [8], we obtain the main result of this paper as follows.

Theorem 9. Let Assumption A hold. Then for each $\epsilon \in(0,1]$, the random dynamical system generated by (23) and (24) possesses a compact random attractor $\mathscr{A}_{\epsilon}$ and the deterministic dynamical system generated by (19) has a global attractor $\mathscr{A}_{0}$ in H. Moreover,

$$
\lim _{\epsilon \rightarrow 0^{+}} \operatorname{dist}_{H}\left(\mathscr{A}_{\epsilon}(\omega), \mathscr{A}_{0}\right)=0
$$

with probability one.

\section{Conflict of Interests}

The author declares that there is no conflict of interests regarding the publication of this paper.

\section{Acknowledgment}

This paper is supported by the special fund of China's central government for the development of local colleges and universities: the project of national first-level discipline in Oil and Gas Engineering.

\section{References}

[1] J. Málek, J. Nečas, M. Rokyta, and M. Růžička, Weak and Measure-Valued Solutions to Evolutionary PDE, vol. 13, Chapman \& Hall, New York, NY, USA, 1996.

[2] O. Ladyzhenskaya, "New equations for the description of the viscous incompressible fluids and solvability in large of the boundary value problems for them," in Boundary Value Problems of Mathematical Physics, American Mathematical Society, Providence, RI, USA, 1970.
[3] H. Bellout, F. Bloom, and J. Nečas, "Young measure-valued solutions for non-Newtonian incompressible fluids," Communications in Partial Differential Equations, vol. 19, no. 11-12, pp. 1763-1803, 1994.

[4] F. Bloom and W. Hao, "Regularization of a non-Newtonian system in an unbounded channel: existence and uniqueness of solutions," Nonlinear Analysis: Theory, Methods \& Applications, vol. 44, pp. 281-309, 2001.

[5] F. Bloom and W. Hao, "Regularization of a non-Newtonian system in an unbounded channel: existence of a maximal compact attractor," Nonlinear Analysis: Theory, Methods \& Applications, vol. 43, no. 6, pp. 743-766, 2001.

[6] C. Guo, B. Guo, and Y. Han, "Random attractors of stochastic non-Newtonian fluids," Acta Mathematicae Applicatae Sinica, vol. 28, no. 1, pp. 165-180, 2012.

[7] M. Pokorný, "Cauchy problem for the non-Newtonian viscous incompressible fluid," Applications of Mathematics, vol. 41, no. 3, pp. 169-201, 1996.

[8] C. Zhao and Y. Li, " $L^{2}$-compact attractor for a non-Newtonian system in two-dimensional unbounded domains," Nonlinear Analysis: Theory, Methods \& Applications, vol. 56, no. 7, pp. 10911103, 2004.

[9] C. Zhao and S. Zhou, " $L^{2}$-compact uniform attractors for a nonautonomous incompressible non-Newtonian fluid with locally uniformly integrable external forces in distribution space," Journal of Mathematical Physics, vol. 48, no. 3, Article ID 032702, 2007.

[10] C. Zhao and J. Duan, "Random attractor for the Ladyzhenskaya model with additive noise," Journal of Mathematical Analysis and Applications, vol. 362, no. 1, pp. 241-251, 2010.

[11] V. V. Chepyzhov and M. I. Vishik, Attractors for Equations of Mathematical Physics, vol. 49 of American Mathematical Society Colloquium Publications, American Mathematical Society, Providence, RI, USA, 2002.

[12] J. K. Hale, Asymptotic behavior of dissipative systems, Mathematical Surveys and Monographs, American Mathematical Society, Providence, RI, USA, 1988.

[13] J. C. Robinson, Infinite-dimensional dynamical systems, Cambridge University Press, Cambridge, UK, 2001.

[14] R. Temam, Infinite Dimensional Dynamical Systems in Mechan$i c s$ and Physics, vol. 68, Springer, Berlin, Germany, 2nd edition, 1997.

[15] L. Arnold, Random Dynamical Systems, Berlin, Germany, Springer, 1998.

[16] G. da Prato and J. Zabczyk, Stochastic Equations in Infinite Dimensions, Encyclopedia of Mathematics and its Applications, Cambridge University Press, Cambridge, 1992.

[17] H. Crauel and F. Flandoli, "Attractors for random dynamical systems," Probability Theory and Related Fields, vol. 100, no. 3, pp. 365-393, 1994.

[18] H. Crauel, A. Debussche, and F. Flandoli, "Random attractors," Journal of Dynamics and Differential Equations, vol. 9, no. 2, pp. 307-341, 1997.

[19] F. Flandoli and B. Schmalfuss, "Random attractors for the $H^{2} \mathrm{D}$ stochastic Navier-Stokes equation with multiplicative white noise," Stochastics and Stochastics Reports, vol. 59, no. 1-2, pp. 21-45, 1996.

[20] T. Caraballo, J. A. Langa, and J. Valero, "Global attractors for multivalued random dynamical systems generated by random differential inclusions with multiplicative noise," Journal of Mathematical Analysis and Applications, vol. 260, no. 2, pp. 602$622,2001$. 
[21] T. Caraballo, J. A. Langa, V. S. Melnik, and J. Valero, "Pullback attractors of nonautonomous and stochastic multivalued dynamical systems," Set-Valued Analysis, vol. 11, no. 2, pp. 153201, 2003.

[22] T. Caraballo, J. A. Langa, and J. Valero, "Global attractors for multivalued random dynamical systems," Nonlinear Analysis: Theory, Methods \& Applications, vol. 48, no. 6, pp. 805-829, 2002.

[23] T. Caraballo and K. Lu, "Attractors for stochastic lattice dynamical systems with a multiplicative noise," Frontiers of Mathematics in China, vol. 3, no. 3, pp. 317-335, 2008.

[24] T. Caraballo, M. J. Garrido-Atienza, B. Schmalfuss, and J. Valero, "Non-autonomous and random attractors for delay random semilinear equations without uniqueness," Discrete and Continuous Dynamical Systems A, vol. 21, no. 2, pp. 415-443, 2008.

[25] T. Caraballo, J. A. Langa, and J. C. Robinson, "Upper semicontinuity of attractors for small random perturbations of dynamical systems," Communications in Partial Differential Equations, vol. 23, no. 9-10, pp. 1557-1581, 1998.

[26] T. Caraballo, J. A. Langa, and J. C. Robinson, "Stability and random attractors for a reaction-diffusion equation with multiplicative noise," Discrete and Continuous Dynamical Systems, vol. 6, no. 4, pp. 875-892, 2000.

[27] T. Caraballo, P. E. Kloeden, and B. Schmalfuß, "Exponentially stable stationary solutions for stochastic evolution equations and their perturbation," Applied Mathematics and Optimization, vol. 50, no. 3, pp. 183-207, 2004.

[28] J. C. Robinson, "Stability of random attractors under perturbation and approximation," Journal of Differential Equations, vol. 186, no. 2, pp. 652-669, 2002. 


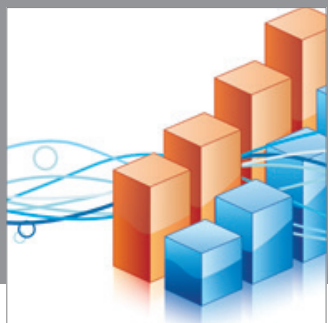

Advances in

Operations Research

mansans

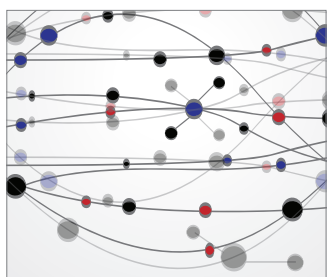

The Scientific World Journal
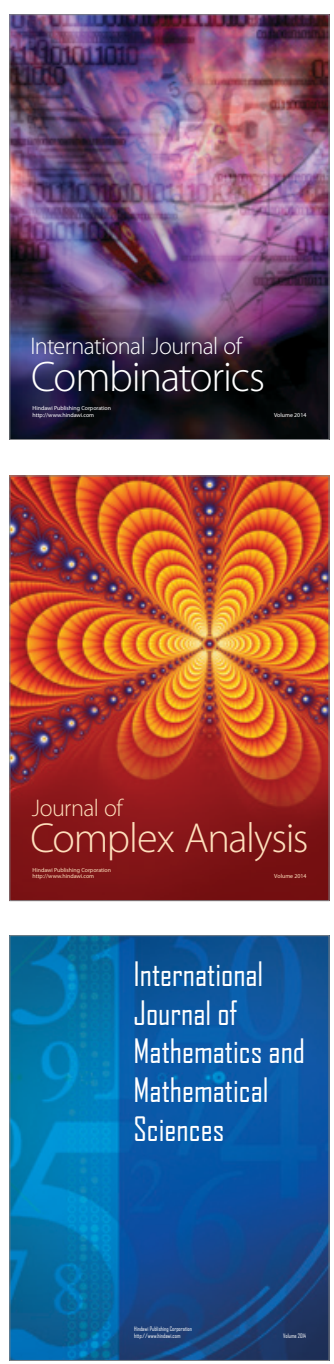
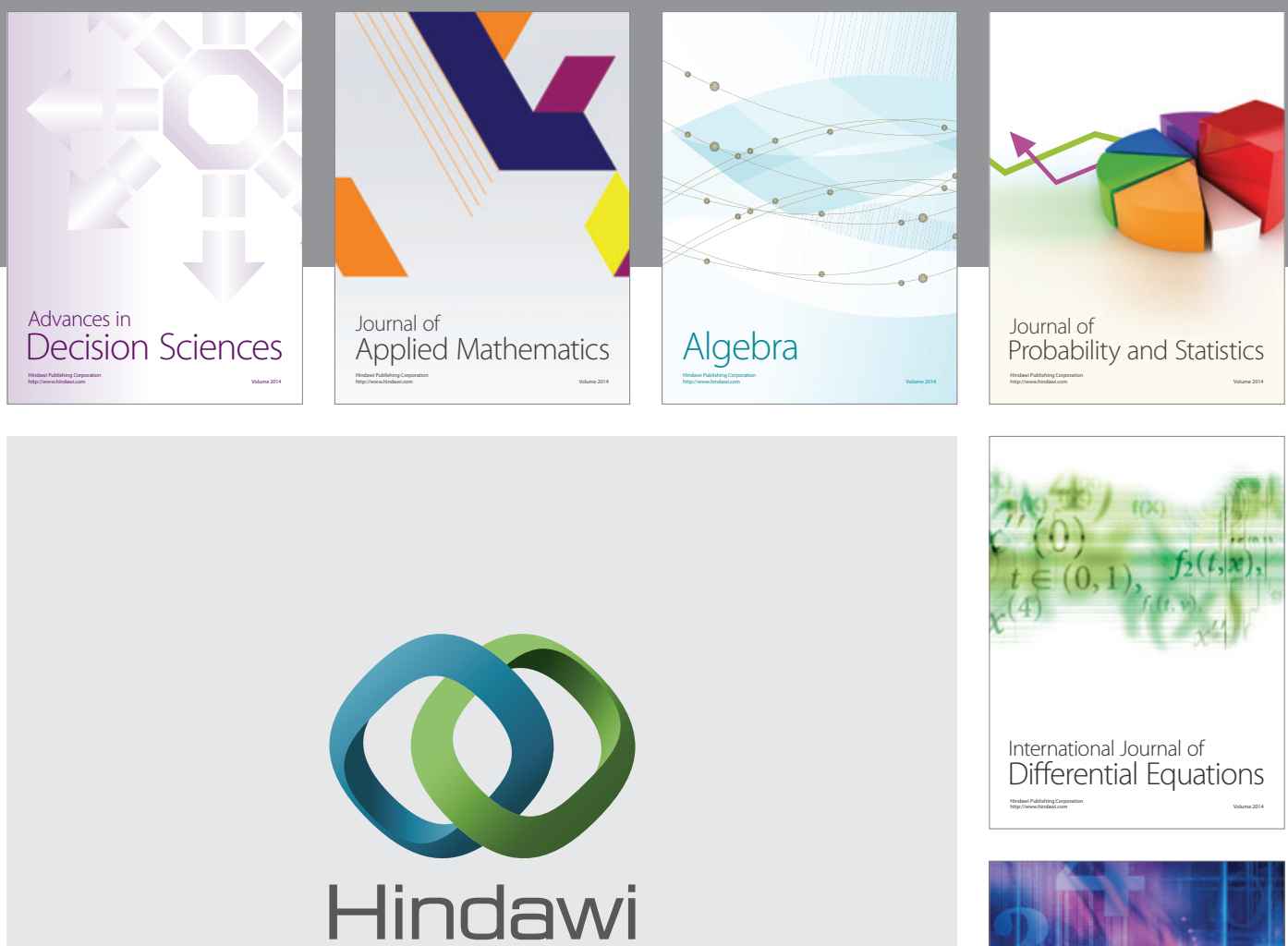

Submit your manuscripts at http://www.hindawi.com
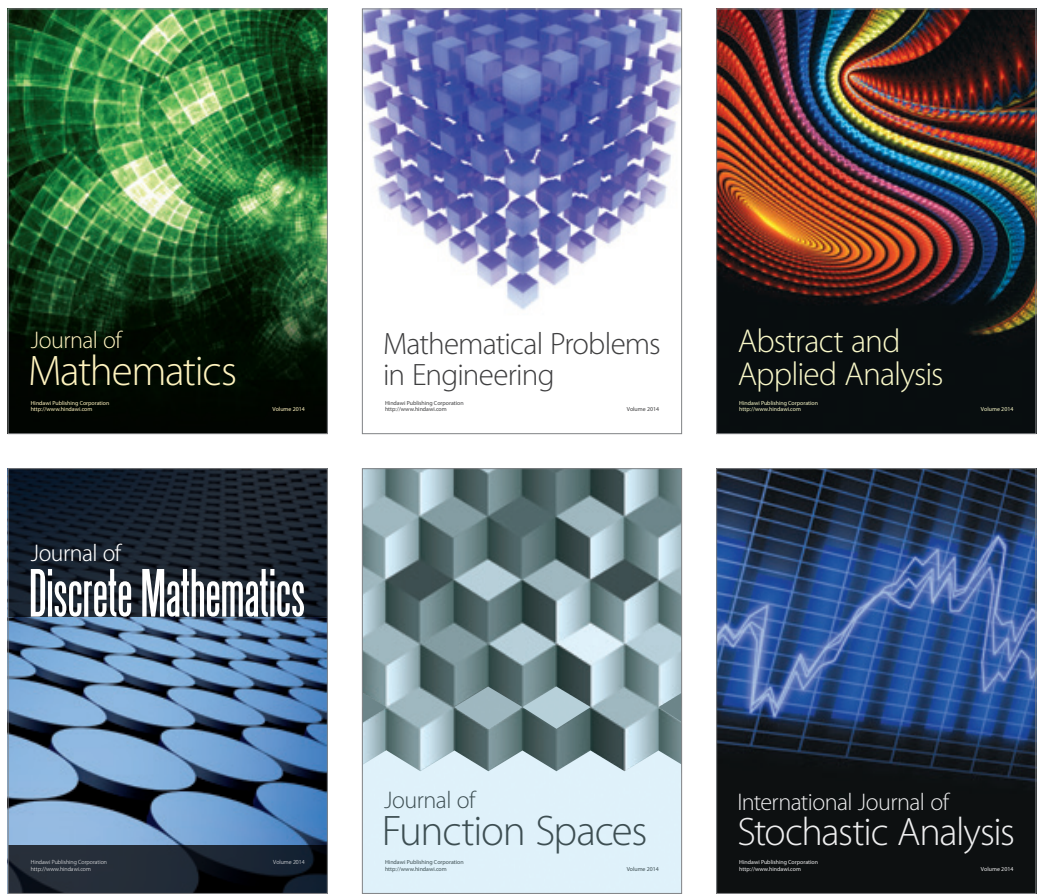

Journal of

Function Spaces

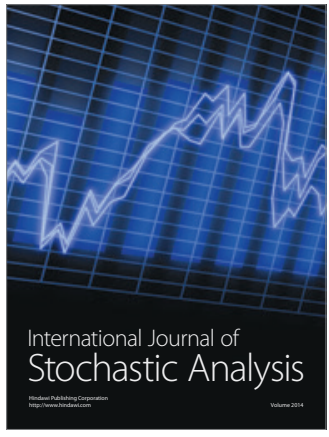

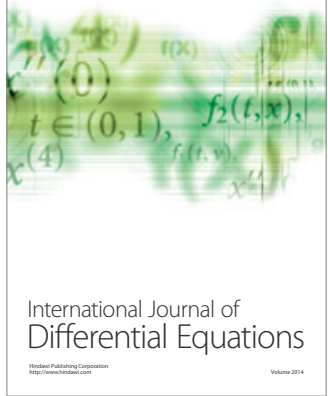
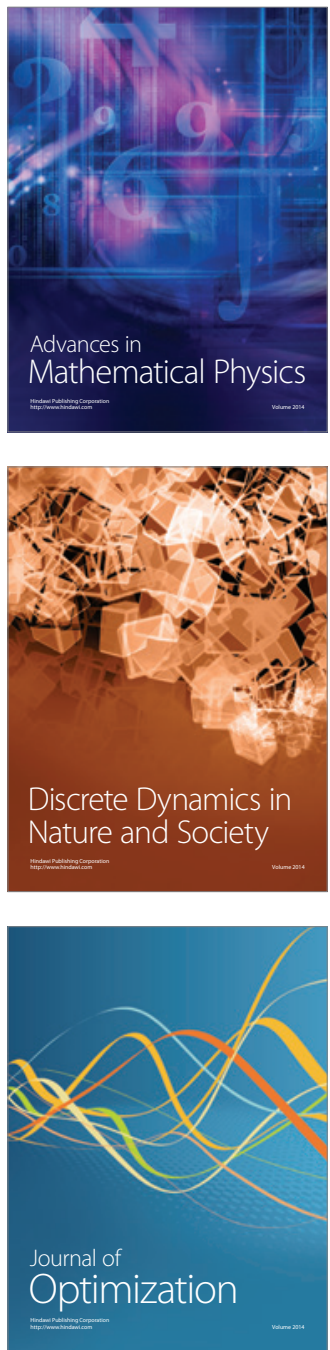